\title{
Erratum to: Choosing Wisely: Prevalence and Correlates of Low- Value Health Care Services in the United States
}

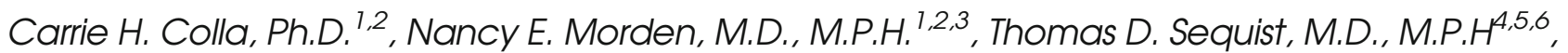
William L. Schpero, M.P.H. ${ }^{7}$, and Meredith B. Rosenthal, Ph.D. ${ }^{7}$

${ }^{1}$ The Dartmouth Institute for Health Policy \& Clinical Practice, Geisel School of Medicine at Dartmouth, Lebanon, NH, USA; ${ }^{2} \mathrm{Norris}$ Cotton Cancer Center, Dartmouth-Hitchcock Medical Center, Lebanon, NH, USA; ${ }^{3}$ Department of Community and Family Medicine, Geisel School of Medicine at Dartmouth, Lebanon, NH, USA; 'Department of Health Care Policy, Harvard Medical School, Boston, MA, USA; ${ }^{5}$ Division of General Internal

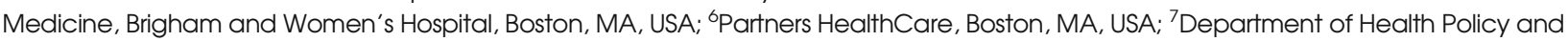
Management, Harvard School of Public Health, Boston, MA, USA.

$\mathrm{J}$ Gen Intern Med 31(4):450

DOI: $10.1007 / \mathrm{s} 11606-015-3420-5$

(c) Society of General Internal Medicine 2015

\section{Erratum to: J Gen Intern Med DOI 10.1007/s11606-014-3070-z}

$\mathrm{O}$ n page 225 of the initial publication, the results for cervical cancer screening in Table 2 mistakenly excluded HCPCS codes G0123, G0124, G0141, G0143-145, G0147, G0148, P3000, P3001 and Q0091. After incorporating these additional codes, the mean annual prevalence of cervical cancer screening increased to $9.8 \%$ from the reported $3.1 \%$. The coefficients in Table 3 and the categorization of HRRs were not meaningfully affected. We regret this error.

Table 2 updates: Low-value diagnostic services: Annual Cervical Cancer Screening: Mean: 9.8\%, Affected National Population (Millions): $14.3 \mathrm{Min} / \mathrm{Max}: 4.3-18.6 \%$, IQR: $8.1 \%-11.0 \%$, COV: 0.24 , Annual Waste: $\$ 23.4 \mathrm{M}$

The online version of the original article can be found at http://dx.doi.org/ 10.1007/s11606-014-3070-z.

Published online July 3, 2015 